\title{
Little gain of function after lower leg revascularization in the elderly
}

Lower extremity revascularization in nursing home residents in the USA is associated with a high mortality and little gain of function at 1 year after surgery. These findings come from a retrospective national cohort study of all US nursing home residents participating in Medicare or Medicaid who underwent this surgery between 2005 and 2009.

A total of 10,784 individuals were included in the study (mean age 82.1 years; $37 \%$ men). Before revascularization, $24.8 \%$ were ambulatory. At 1 year follow-up, $51 \%$ of the patients had died, $28 \%$ were nonambulatory, and $32 \%$ had declined in functional status. Only $13 \%$ of the initial cohort were ambulatory, and $18 \%$ had maintained or improved their functional status. Residents who were ambulatory before surgery were more likely to have better outcomes than those who were nonambulatory; however, revascularization rarely restored ambulatory function in a previously nonambulatory patient. In a multivariate analysis, age $\geq 80$ years, cognitive impairment, and heart or kidney failure were associated with poor prognosis.

The investigators conclude that " $\mathrm{a}$ substantial number of nursing home residents in the United States undergo lower extremity revascularization, and many gain little, if any, function". Nevertheless, "ambulatory function, although clearly an important goal, may not be the primary objective of treatment". Patients who undergo revascularization can also benefit from pain relief, wound healing, and avoidance of major amputation, and the results of this study must be interpreted in this context.

Gregory B. Lim

Original article Oresanya, L. et al. Functional outcomes after lower extremity revascularization in nursing home residents: a national cohort study. JAMA Intern. Med. doi:10.1001/jamainternmed.2015.0486 